\title{
Avaliação de fatores de ambiente e estimativas de parâmetros genéticos para a característica dias para o parto na raça Nelore ${ }^{1}$
}

\author{
Selma Forni' ${ }^{2}$, Lucia Galvão de Albuquerque ${ }^{3}$ \\ ${ }^{1}$ Parte da dissertação de Mestrado em Zootecnia da primeira autora apresentada à FCAV/UNESP - Jaboticabal/SP. Projeto financiado \\ pela FAPESP. \\ 2 Doutoranda em Zootecnia da FCAVIUNESP - Jaboticabal/SP. Bolsista CNPq. \\ ${ }^{3}$ Departamento de Zootecnia da FCAVIUNESP - Jaboticabal/SP. Bolsista CNPq.
}

RESUMO - Dados de um rebanho comercial da raça Nelore foram analisados com os objetivos de avaliar a influência de fatores ambientais e estimar parâmetros genéticos para a característica dias para o parto (DPP). Foram utilizados modelos fixos que incluíram os efeitos de grupo de contemporâneos, sexo do bezerro, idade da vaca no início da estação de monta, mês do parto anterior e peso do bezerro desmamado no final da estação de monta (apenas este último efeito não influenciou a característica significativamente). Duas definições de grupo de contemporâneos foram consideradas: a primeira definida pelas informações fazenda, ano e estação de monta, grupos de manejo (nascimento, desmama e reprodução) e tipo de serviço (monta natural, monta controlada ou inseminação artificial); e a segunda, pelas mesmas informações mais o sexo do bezerro. As análises genéticas foram realizadas com conjuntos de dados que incluíram ou não os animais sem registros de parto. Quando incluídos, o valor de DPP atribuído a esses animais foi igual ao maior valor observado dentro do grupo de manejo somado a 21 dias. Os componentes de variância foram estimados por máxima verossimilhança restrita utilizando-se modelos animal unicaracterística. A inclusão do efeito aleatório de ambiente permanente nos modelos foi avaliada mediante o "Likelihood Ratio Test". As herdabilidades estimadas variaram entre 0,01 e 0,11 e a inclusão do efeito de ambiente permanente nos modelos foi significativa, de modo que a exclusão deste efeito superestimou a variância genética aditiva. A metodologia aplicada para penalizar os animais que não pariram não melhorou a identificação das diferenças genéticas entre eles. Os resultados indicaram que, como grande parte das características reprodutivas em bovinos, a DPP sofre grande influência do ambiente.

Palavras-chave: características reprodutivas, efeitos ambientais, gado de corte, parâmetros genéticos

\section{Environmental effects and genetic parameters estimates for days to calving in Nelore cattle}

\begin{abstract}
Data from a Nelore population was used to evaluate environmental effects and to estimate genetic parameters for days to calving (DPP). The influence of contemporary group, age at breeding season, month of last calving and weaning weight of the calf at the end of the breeding season were evaluated with fixed models. The weaning weight of the calf at the end of the breeding season was the only effect that did not influence DPP. Two different contemporary group (CG) definitions were also evaluated. Herd, year and season of breeding, management groups (birth, weaning and breeding) and mating type (multiple sires, single sire or artificial insemination) defined the first CG and the second CG included the same variables and sex of the calf. Variance components were estimated using restricted maximum likelihood method fitting univariate animal models, including or not non-calvers cows (which were penalized by adding 21 days to the largest DPP record in their management group). Heritability estimates ranged between 0.01 and 0.11 . Exclusion of the permanent environmental effect from the model overestimated additive genetic variance. The method of assigning penalties to non-calvers cows was unsuitable; it resulted in reduced additive genetic variance. The results suggest that DPP is strongly influenced by the environment.
\end{abstract}

Key Words: beef cattle, environmental effects, genetic parameters, reproductive traits

\section{Introdução}

O desempenho reprodutivo dos animais é um dos principais fatores determinantes da eficiência total de produção na bovinocultura de corte. Cada vez mais precocidade sexual e regularidade reprodutiva são características desejáveis em um rebanho de corte. Entre os objetivos em diversos programas de seleção, a precocidade sexual tem se destacado por permitir que o criador ganhe tempo expondo animais mais jovens à reprodução, diminuindo o intervalo de gerações. Além disso, o processo reprodutivo influencia o progresso genético em outras características, pois a pressão de seleção exercida depende, entre outros fatores, do desempenho reprodutivo do rebanho. Entretanto, como 
reconhecido por Notter \& Johnson (1988), as características reprodutivas são de mais difícil medição, registro e interpretação e os procedimentos disponíveis para predição dos méritos genéticos dos animais, na maioria dos casos, não são diretos.

A característica dias para o parto (DPP), calculada como o intervalo, em dias, do primeiro dia da estação de monta à data do parto subseqüente, tem sido apontada como alternativa para se avaliar a fertilidade das fêmeas, especialmente em sistemas extensivos. Esta característica reflete a variabilidade das fêmeas em apresentar atividade de estro durante a estação de monta, o número de serviços requeridos para a prenhez, a duração da gestação e, conseqüentemente, a data do parto. Permite ainda a identificação dos animais com maior fertilidade dentro do rebanho, das fêmeas que emprenham mais precocemente dentro da estação de monta e dos touros que produzem filhas que emprenham mais cedo dentro da estação de monta.

Objetivou-se com este trabalho avaliar a influência de fatores ambientais e estimar parâmetros genéticos para a característica DPP em uma população de animais da raça Nelore.

\section{Material e Métodos}

Os dados utilizados neste trabalho foram fornecidos pela Agropecuária Jacarezinho Ltda., situada em Valparaíso, noroeste do estado de São Paulo. A empresa dedica-se à criação de bovinos de corte, predominantemente da raça Nelore, em sistema extensivo. Os animais são periodicamente avaliados e selecionados e as pesagens ocorrem ao nascimento, à desmama (aproximadamente 205 dias de idade) e ao sobreano (aproximadamente 550 dias de idade). A avaliação de "CPMU", na qual se atribuem aos animais notas de 1 a 5 quanto à conformação $(\mathrm{C})$, à precocidade $(\mathrm{P})$, à musculatura $(\mathrm{M})$ e ao umbigo $(\mathrm{U})$, é realizada durante as pesagens à desmama e ao sobreano e a circunferência escrotal é medida apenas nesta última idade. A seleção é realizada com base em índices compostos pelas características avaliadas em cada período (desmama e sobreano), classificando-se os animais, de acordo com seus índices, em decas, em que deca 1 representa os $10 \%$ melhores animais.

À desmama, são descartadas as fêmeas da deca 10 $(10 \%)$ e os machos das decas 6 a $10(50 \%)$. Ao sobreano repete-se a mesma pressão de seleção e os machos das decas 1 a 5 são submetidos a exames andrológicos para serem vendidos ou utilizados como reprodutores. Portanto, aproximadamente $81 \%$ das fêmeas nascidas na propriedade permanecem no rebanho para se tornarem matrizes e $25 \%$ dos machos se tornam reprodutores. A cada ano, os 15-20 melhores tourinhos são submetidos a teste de progênie.
A maior parte dos acasalamentos ocorre por monta natural em duas estações de monta distintas: a primeira, com início em fevereiro, tem duração de aproximadamente 60 dias, período em que apenas novilhas de 16 a 18 meses são expostas à monta; a segunda, com início no mês de novembro, tem duração aproximada de 75 dias, período único em que todas as fêmeas têm oportunidade de emprenhar, incluindo aquelas que participaram da primeira estação e falharam. A descrição mais detalhada sobre o manejo e o programa de melhoramento genético da Agropecuária Jacarezinho pode ser encontrada em Gava Filho (1997).

$\mathrm{O}$ arquivo de dados original continha 100.282 registros de nascimentos ocorridos no período de 1984 a 2000 . Na primeira verificação da consistência dos dados, foram eliminados registros com informações incompletas e aqueles muito distantes da realidade biológica, como registros de DPP menores que o período de gestação, admitido como mínimo. Assim, o arquivo criado para avaliação dos efeitos ambientais e para as análises genéticas, com todos os registros de fêmeas que pariram pelo menos uma vez, continha dados de identificação do animal, data de nascimento, pai, mãe, tipo de serviço e grupos de manejo (nascimento, desmama e sobreano/reprodução), data do parto, sexo e peso do bezerro. Esse arquivo era composto de 53.181 registros de partos, nos quais 43.094 foram obtidos por acasalamento por monta natural, 6.187 por inseminação artificial e 3.900 por monta controlada. A formação e edição dos arquivos foram feitas pelo programa computacional SAS (SAS, 1998).

Foram avaliados os efeitos de grupo de contemporâneos, sexo do bezerro, idade da vaca no início da estação de monta, mês do parto anterior e peso do bezerro desmamado no final da estação de monta sobre a expressão da característica DPP. A definição de grupo de contemporâneos e os demais efeitos fixos foram adotados, visto que estudos anteriores comprovaram efeito significativo dessas variáveis sobre DPP (Meyer et al., 1990; Meyer et al., 1991; Ponzoni \& Gifford, 1994; Johnston \& Bunter, 1996; Bergmann et al., 1998; Gressler et al., 2000; Mercadante, 2001; Pereira, 2001).

Dois grupos de contemporâneos foram considerados: o primeiro (GC1) com informações de fazenda, ano e estação de monta, grupos de manejo (nascimento, desmama e reprodução) e tipo de serviço (monta natural, monta controlada ou inseminação artificial); e o segundo (GC2) com as mesmas variáveis que o primeiro mais o sexo do bezerro. Foram eliminados dos arquivos os grupos com menos de quatro animais, quando avaliada a $\mathrm{GC} 1$, e com menos de três animais, quando avaliada a GC2. A média de registros de DPP por grupo de contemporâneos, antes da inclusão do sexo do bezerro (GC1), foi de 16 observações, sendo que 
$52 \%$ dos registros ficaram em grupos com mais de dez observações. Com a inclusão do sexo do bezerro (GC2), a média de registros de DPP por grupo de contemporâneos passou a ser de 9,6 observações e diminuiu para $32 \%$ o número de grupos com mais de dez observações.

$\mathrm{O}$ peso à desmama do bezerro amamentado durante a estação de monta foi avaliado como efeito fixo classificatório, de modo que, em cada classe, foram agrupados animais com diferenças de peso de até $5 \mathrm{~kg}$. Para os registros de fêmeas de primeira cria, foi criada uma classe única, pois não existe o efeito do bezerro sobre o primeiro registro de DPP. O mesmo procedimento foi adotado para avaliação do efeito do mês do parto anterior. $\mathrm{Na}$ análise que incluiu o efeito do peso do bezerro desmamado no final da estação de monta, o número de grupos de contemporâneos diminuiu de 4.809 para 4.774, em virtude da eliminação de 618 observações de parto (não havia registro do peso à desmama do bezerro nascido no parto anterior), entretanto, a média de observações por grupo de contemporâneos não foi alterada.

As análises estatísticas foram realizadas utilizando-se o método dos quadrados mínimos, via modelos fixos, por meio do procedimento GLM do programa SAS (SAS, 1998), que podem ser representados da seguinte forma:

Modelo 1: $\mathrm{y}_{\mathrm{ijl}}=\mu+\mathrm{GCl}_{\mathrm{i}}+\mathrm{SB}_{\mathrm{j}}+\mathrm{b}_{1}\left(\mathrm{IDV}_{\mathrm{ijl}}-\mathrm{IDV}\right)+$ $\mathrm{b}_{2}\left(\mathrm{IDV}_{\mathrm{ijl}}-\mathrm{IDV}\right)^{2}+\mathrm{e}_{\mathrm{ij} 1}$

Modelo 2: $\mathrm{y}_{\mathrm{il}}=\mu+\mathrm{GC}_{\mathrm{i}}+\mathrm{b}_{1}\left(\mathrm{IDV}_{\mathrm{il}}-\mathrm{IDV}\right)+\mathrm{b}_{2}\left(\mathrm{IDV}_{\mathrm{il}}\right.$ - IDV) $)^{2}+\mathrm{e}_{\mathrm{il}}$

Modelo 3: $\mathrm{y}_{\mathrm{ikl}}=\mu+\mathrm{GC2}_{\mathrm{i}}+\mathrm{MPA}_{\mathrm{k}}+\mathrm{b}_{1}\left(\mathrm{IDV}_{\mathrm{ikl}}-\mathrm{IDV}\right)$ $+\mathrm{b}_{2}\left(\mathrm{IDV}_{\mathrm{ikl}}-\mathrm{IDV}\right)^{2}+\mathrm{e}_{\mathrm{ikl}}$

Modelo 4: $\mathrm{y}_{\mathrm{ikml}}=\mu+\mathrm{GC}_{\mathrm{i}}+\mathrm{MPA}_{\mathrm{k}}+\mathrm{PDB}_{\mathrm{m}}+\mathrm{b}_{1}$ $\left(\mathrm{IDV}_{\mathrm{ikml}}-\mathrm{IDV}\right)+\mathrm{b}_{2}\left(\mathrm{IDV}_{\mathrm{ikml}}-\mathrm{IDV}\right)^{2}+\mathrm{e}_{\mathrm{ikml}}$

em que: $y_{\mathrm{ijl}}=$ dias para o parto do animal l, pertencente ao iésimo grupo de contemporâneos, que pariu um bezerro do jésimo sexo; $y_{\mathrm{il}}=$ dias para o parto do animal 1 , pertencente ao iésimo grupo de contemporâneos; $\mathrm{y}_{\mathrm{ikl}}=$ dias para o parto do animal l, pertencente ao iésimo grupo de contemporâneos, que teve seu parto anterior no $\mathrm{k}^{\text {ésimo }}$ mês; $\mathrm{y}_{\mathrm{ikml}}=$ dias para o parto do animal 1 , pertencente ao iésimo grupo de contemporâneos, que teve seu parto anterior no $\mathrm{k}^{\text {ésimo }}$ mês, que desmamou um bezerro da $\mathrm{m}^{\text {ésima }}$ classe de peso; $\mu=$ média geral das medidas de dias para o parto; $\mathrm{GC}_{\mathrm{i}}=$ efeito do i ésimo grupo de contemporâneos; $\mathrm{SB}_{\mathrm{j}}=$ efeito do jésimo sexo do bezerro; $\mathrm{MPA}_{\mathrm{k}}=$ efeito do $\mathrm{k}^{\text {ésimo }}$ mês do parto anterior; $\mathrm{PDB}_{\mathrm{m}}=$ efeito da $\mathrm{m}^{\text {ésima }}$ classe de peso do bezerro desmamado no final da estação de monta; $\operatorname{IDV}_{\mathrm{ijl}}=$ idade no início da estação de monta do animal l, pertencente ao iésimo grupo de contemporâneos, que pariu um bezerro do jésimo sexo; $I_{\text {il }}=$ idade no início da estação de monta do animal 1, pertencente ao iésimo grupo de contemporâneos; $\mathrm{IDV}_{\mathrm{ikl}}=$ idade no início da estação de monta do animal l, pertencente ao i ${ }^{\text {ésimo }}$ grupo de contemporâneos, que teve seu parto anterior no $\mathrm{k}^{\text {ésimo }}$ mês; $\mathrm{IDV}_{\mathrm{ikml}}=$ idade no início da estação de monta do animal 1, pertencente ao iésimo grupo de contemporâneos, que teve seu parto anterior no $\mathrm{k}^{\text {ésimo }}$ mês, que desmamou um bezerro da $\mathrm{m}^{\text {ésima }}$ classe de peso; IDV = média da idade da vaca no início da estação de monta; $b_{1}$ e $b_{2}=$ coeficientes de regressão linear e quadrático da idade da vaca no início da estação de monta; $\mathrm{e}_{\mathrm{ij} 1}, \mathrm{e}_{\mathrm{il}}, \mathrm{e}_{\mathrm{ikl}} \mathrm{e}$ $\mathrm{e}_{\mathrm{ikml}}=$ erros aleatórios associados às observações;

Para a análise genética, foram empregados três modelos unicaracterística, definidos a partir dos resultados das análises estatísticas preliminares, com os diferentes efeitos fixos, considerando-se aleatórios os efeitos genético direto (animal) e de ambiente permanente. O modelo geral utilizado pode ser representado na forma matricial como:

$$
y=X \beta+Z_{1} a+Z_{2} c+e
$$

em que: $y=$ vetor da variável dependente; $\beta=$ vetor de efeitos fixos

Modelo 1: GC1, SB e IDV (efeitos linear e quadrático);

Modelo 2: GC2 e IDV (efeitos linear e quadrático);

Modelo 3: GC2, MPA e IDV (efeitos linear e quadrático);

$a=$ vetor de efeitos genéticos aditivos; $c=$ vetor de efeitos de ambiente permanente; $e=$ vetor de efeitos residuais; $X \mathrm{e}$ $Z$ são as matrizes de incidência respectivas para cada efeito.

Pressuposições:

$$
\mathrm{E}(y)=X \beta, \mathrm{E}(a)=0, \mathrm{E}(c)=0 \mathrm{e} \mathrm{E}(e)=0, \mathrm{e}
$$

$$
\operatorname{Var}\left[\begin{array}{l}
a \\
c \\
e
\end{array}\right]=\left[\begin{array}{lll}
G & 0 & 0 \\
0 & C & 0 \\
0 & 0 & R
\end{array}\right]
$$

em que: $G=A \sigma_{a}^{2}$, matriz de variância genética aditiva; $A=$ matriz de parentesco; $\sigma_{a}^{2}=$ variância genética aditiva; $C=I \sigma_{c}^{2}$, matriz de variância decorrente do efeito de ambiente permanente; $I=$ matriz de identidade; $\sigma_{c}^{2}=$ variância atribuída ao efeito de ambiente permanente; $R=I \sigma_{e}^{2}$, matriz de variância residual; $\sigma_{e}^{2}=$ variância residual.

Os componentes de variância foram estimados por máxima verossimilhança restrita, utilizando-se o programa 
MTDFREML - Multiple Trait Derivative Free Restricted Maximum Likelihood (Boldman et al., 1993). Considerou-se que o critério de convergência foi alcançado quando a variância dos valores do logaritmo da função de verossimilhança foi igual ou menor que $10^{-9}$. Como há possibilidade de convergência em máximos locais (Pres et al., 1986), as análises foram repetidas para assegurar a convergência no máximo global da função de verossimilhança, de modo que, a cada interação, os valores iniciais dos componentes de variância foram obtidos da análise anterior. A inclusão do efeito de ambiente permanente nos modelos foi avaliada mediante o Likelihood Ratio Test (Hogg \& Craig, 1995), comparando-se os valores dos logaritmos das funções de máxima verossimilhança obtidos em análises com modelos que incluíam ou não este efeito.

Também foram realizadas análises incluindo todas as fêmeas expostas à reprodução, mesmo aquelas sem nenhum parto notificado, visto que Meacham \& Notter, (1987), Notter \& Johnson (1988) e Meyer et al. (1990) relataram que a exclusão destas fêmeas da avaliação de características reprodutivas pode mascarar a variabilidade e as diferenças genéticas entre os animais. Aos animais que não pariram foi atribuída a penalização descrita por Johnston \& Bunter (1996). O DPP desses animais foi definido somando-se 21 dias ao mais alto valor encontrado no grupo de manejo, definindo-se o grupo de manejo pela combinação das informações: grupos de manejo do nascimento, da desmama e do sobreano, fazenda, ano e estação da monta.

Nas análises incluindo os animais que não pariram, foram avaliadas 70.154 observações de 43.607 fêmeas em 6.391 grupos de contemporâneos (GC2). A análise genética foi realizada empregando-se modelos animal unicaracterísticos que continham os efeitos fixos de grupo de contemporâneos, mês do parto anterior e idade da vaca no início da estação de monta como covariável (linear e quadrático). No primeiro modelo, foi incluído como efeito aleatório apenas o efeito genético aditivo e, no segundo modelo, foi incluído, além do efeito genético aditivo, o efeito de ambiente permanente.

\section{Resultados e Discussão}

A média e o desvio-padrão observados para a característica DPP foram, respectivamente, 306 e 25 dias, semelhantes aos obtidos com a raça Angus por Johnston \& Bunter (1996), de 304 e 29 dias. Ressalta-se que esses valores são altamente influenciados pelo manejo adotado no rebanho em questão, especialmente pela duração da estação de monta, e que a pequena variação encontrada
$(8,27 \%)$ decorre da própria definição da característica que envolve a soma do período de gestação com um valor que será, no máximo, a duração da estação de monta. Considerando o período médio de gestação da raça Nelore, 290 dias (Ferraz \& Eler, 1998; Pereira et al., 2000), o tempo médio préconcepção na estação de monta (período de serviço) foi de, aproximadamente, 16 dias.

Os efeitos fixos de grupo de contemporâneos ( $\mathrm{GCl}$ e GC2), sexo do bezerro, idade da vaca no início da estação de monta e mês do parto anterior foram importantes fontes de variação para a característica DPP (Tabela 1).

$\mathrm{O}$ efeito do sexo do bezerro resultou em acréscimo de aproximadamente 1,9 dias nos valores de DPP, que foram maiores nas fêmeas que gestaram machos. Esse efeito pode estar associado ao aumento no período de gestação, atribuído ao sexo e à duração da gestação de machos, e tende a ser maior conforme aumenta o peso ao nascer (Bourdon \& Brinks, 1982; Pereira, 2001). Bergmann et al. (1998) observaram que, para cada quilo a mais no peso ao nascer do bezerro, houve aumento de 2,4 dias na data juliana do primeiro parto.

O peso do bezerro desmamado ao final da estação de monta não influenciou significativamente o DPP, indicando que o fato de as fêmeas estarem amamentando durante a estação reprodutiva não prejudicou seu desempenho. Resultados semelhantes foram descritos por Johnston \& Bunter (1996), que compararam animais que amamentaram ou não durante a estação de monta, separando-os em diferentes classes de idade, e verificaram aumento muito pequeno ( 0,3 dias) nos valores de DPP daqueles que estavam amamentando. Os autores concluíram que, para a avaliação genética, não seria necessário ajustar os valores de DPP em função do estádio de lactação das vacas Angus.

Os animais que tiveram seu último parto em meses mais próximos aos de início da estação de monta apresentaram maiores valores de DPP, evidenciando que fêmeas que emprenham no início da estação reprodutiva e parem no início da estação de parição entram em melhores condições na estação de monta seguinte e têm maiores chances de emprenhar mais precocemente. Pela observação das soluções mínimo-quadráticas para o efeito do mês do parto anterior, verifica-se que as novilhas precoces que emprenharam na estação de monta do mês de fevereiro e tiveram intervalo da 1 a à $2^{a}$ estação de monta superior a 12 meses apresentaram DPP de 3 a 11 dias menores que o das demais fêmeas.

Constam na Figura 1 os valores de DPP estimados em função da idade do animal no início da estação de monta considerando os demais fatores ambientais que afetam a 
Tabela 1 - Análise de variância da característica dias para o parto de animais da raça Nelore

Table 1 - Analysis of variance of days to calving for Nelore beef cattle

\begin{tabular}{lcc}
\hline $\begin{array}{l}\text { Fonte de variação } \\
\text { Source of variation }\end{array}$ & $\begin{array}{c}\text { Graus de liberdade } \\
\text { Degrees of freedom }\end{array}$ & $\begin{array}{c}\text { Quadrado médio } \\
\text { Mean square }\end{array}$ \\
\hline Modelo 1 & & \\
Model 1 & & $6.192,18^{* *}$ \\
\hline GC1 & 2.916 & $42.459,11^{* *}$ \\
SB & 1 & $4.981,05^{* *}$ \\
IDV & 1 & 40,05 \\
IDV 2 & 1 & 283,31 \\
Resíduo & 44.635 & \\
Residual & & \\
$* *(\mathrm{P}<0,01), \mathrm{R}^{2}=0,5907$ & & \\
\hline
\end{tabular}

Modelo 2

Model 2

\begin{tabular}{lcc}
\hline GC2 & 4.808 & $3.685,70^{* *}$ \\
$\mathrm{IDV}$ & 1 & $3.162,27^{* *}$ \\
$\mathrm{IDV} 2$ & 1 & 38,83 \\
Resíduo & 41.359 & 284,27 \\
Residual & & \\
$* *(\mathrm{P}<0,01), \mathrm{R}^{2}=0,6033$ & & \\
\hline
\end{tabular}

Modelo 3

Model 3

\begin{tabular}{lcc}
\hline $\mathrm{GC} 2$ & 4.808 & $3.689,02 * *$ \\
$\mathrm{MPA}$ & 8 & $39.523,42 * *$ \\
$\mathrm{IDV}$ & 1 & $38.171,72^{* *}$ \\
$\mathrm{IDV}{ }^{2}$ & 1 & $14.975,15 * *$ \\
Resíduo & 41.362 & 276,75 \\
Residual & & \\
$* *(\mathrm{P}<0,01), \mathrm{R}^{2}=0,6138$ & & \\
\hline
\end{tabular}

Modelo 4

Model 4

\begin{tabular}{lcc}
\hline GC2 & 4.773 & $3.649,97^{* *}$ \\
MPA & 8 & $39.582,08^{* *}$ \\
PDB & 25 & 164,31 \\
IDV & 1 & $39.008,40^{* *}$ \\
IDV 2 & 1 & $15.419,73^{* *}$ \\
Resíduo & & \\
Residual & 40.688 & 276,93 \\
$* *(P<0,01), R^{2}=0,6139$ & & \\
\hline
\end{tabular}

$\mathrm{GC}=$ grupo de contemporâneos, $\mathrm{SB}=$ sexo do bezerro, IDV = efeito linear da idade da vaca no início da estação de monta, IDV ${ }^{2}=$ efeito quadrático da idade da vaca no início da estação de monta, MPA = mês do parto anterior, $\mathrm{PDB}=$ peso do bezerro desmamado no final da estação de monta, $\mathrm{R}^{2}=$ coeficiente de determinação.

$G C=$ contemporary group, $S B=$ sex of calf,$I D V=$ linear effect of dam age at breeding season, $I D V^{2}=$ quadratic effect of dam age at breeding season, MPA = month of last calving, $P D B=$ weaning weight at the end of the breeding season, $R^{2}=$ coefficient of determination.

expressão de DPP (Modelo 3). Os valores estimados tenderam a diminuir com o aumento da idade do animal até aproximadamente quatro anos, de modo que, quando a idade aumentou de 1 para 2 anos, os valores diminuíram aproximadamente dois dias e, quando a idade aumentou de dois para quatro anos, os valores de DPP tenderam a diminuir um dia para cada ano. Johnston \& Bunter (1996) avaliaram o efeito da idade da vaca na estação de monta separando os animais em três grupos de idade (o primeiro

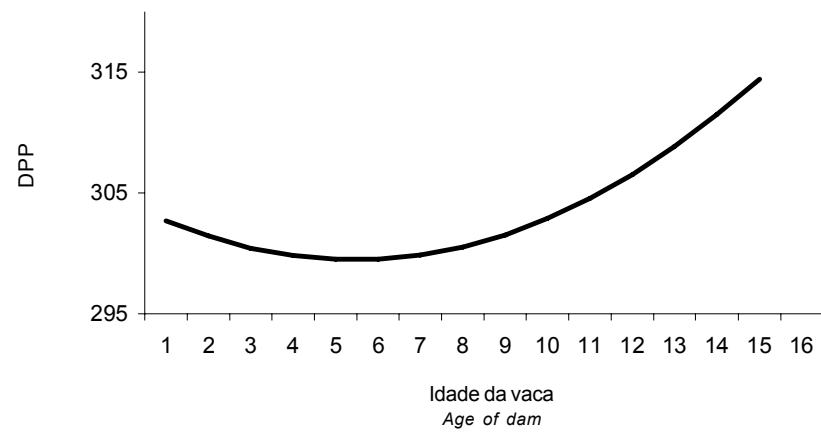

Figura 1 - Efeito da idade da vaca no início da estação de monta sobre os valores estimados de dias para o parto (DPP).

Figure 1 - Effect of age of dam at breeding season on days to calving estimates (DPP).

com fêmeas de um ano, o segundo com fêmeas de dois anos de idade e o terceiro com animais adultos, de três e quatro anos) e também verificaram tendência de redução dos valores de DPP com o aumento da idade do animal. Neste trabalho, a tendência de queda dos valores estimados de DPP no início da curva pode ter sido influenciada pelo descarte anual de todas as fêmeas que não emprenharam; as fêmeas mais velhas são aquelas que permaneceram na fazenda após rigoroso processo de seleção e não falharam em nenhuma das vezes que foram expostas à monta.

Na Figura 1 verifica-se também forte tendência de aumento dos valores de DPP com o avançar da idade a partir dos sete anos. Pereira (2001) observou aumento no período de gestação com o aumento da idade de animais com mais de sete anos. Esse aumento pode contribuir para maiores DPP, entretanto, apenas $10 \%$ dos registros avaliados neste trabalho são de fêmeas que entraram na estação de monta com sete anos ou mais.

A inclusão dos diferentes efeitos fixos, ainda que significativos, não provocou grandes alterações nos coeficientes de determinação dos modelos. Entretanto, para a avaliação genética dos animais, esses efeitos devem ser considerados por constituírem-se importantes fontes de variação e porque a definição de grupo de contemporâneos utilizada deve contemplar as interações que atuam significativamente na expressão da característica sem reduzir demasiadamente a média de observações por grupo.

As estimativas dos componentes de variância e herdabilidade obtidas são apresentadas na Tabela 2. A inclusão do efeito de ambiente permanente do animal no modelo aumentou significativamente o valor do logaritmo da função de máxima verossimilhança. É possível observar que 6,3 a $6,9 \%$ da variância fenotípica pode ser atribuída a este efeito e que sua exclusão, por ocasião da estimação dos 
Tabela 2 - Estimativas de componentes de variância para a característica dias para o parto (DPP) em bovinos da raça Nelore Table 2 - Variance components estimates for days to calving in Nelore beef cattle

\begin{tabular}{|c|c|c|c|c|c|c|}
\hline $\begin{array}{l}\text { Modelo } \\
\text { Model }\end{array}$ & $\sigma_{a}^{2}$ & $\sigma_{\mathrm{c}}^{2}$ & $\sigma_{\mathrm{e}}^{2}$ & $\mathrm{~h}^{2} \pm \mathrm{EP}$ & $c^{2} \pm E P$ & $\mathrm{e}^{2} \pm \mathrm{EP}$ \\
\hline 1 & 31,58 & - & 252,76 & $0,11 \pm 0,005$ & - & $0,89 \pm 0,005$ \\
\hline 1 & 13,72 & 19,58 & 250,80 & $0,05 \pm 0,012$ & $0,069 \pm 0,013$ & $0,88 \pm 0,005$ \\
\hline 2 & 28,65 & - & 256,71 & $0,10 \pm 0,005$ & - & $0,90 \pm 0,005$ \\
\hline 2 & 12,79 & 19,62 & 252,52 & $0,04 \pm 0,009$ & $0,069 \pm 0,010$ & $0,89 \pm 0,006$ \\
\hline 3 & 23,31 & - & 254,77 & $0,08 \pm 0,005$ & - & $0,92 \pm 0,005$ \\
\hline 3 & 11,00 & 14,51 & 249,67 & $0,04 \pm 0,008$ & $0,063 \pm 0,010$ & $0,90 \pm 0,006$ \\
\hline
\end{tabular}

$\sigma^{2}{ }_{a}=$ variância genética aditiva, $\sigma^{2}=$ variância atribuída ao efeito aleatório de ambiente permanente, $\sigma^{2}{ }_{e}=$ variância residual, $h^{2} \pm E P=$ herdabilidade \pm erropadrão, $c^{2} \pm E P=$ fração da variância fenotípica atribuída ao ambiente permanente \pm erro-padrão, $e^{2} \pm E P=$ fração da variância fenotípica atribuída ao ambiente temporário \pm erro-padrão.

$\sigma_{a}^{2}=$ additive genetic variance, $\sigma_{c}^{2}=$ permanent environment variance, $\sigma_{e}^{2}=$ residual variance, $h^{2} \pm E P=$ heritability \pm standard error, $c^{2} \pm E P=$ fraction of phenotypic variance due to permanent environment \pm standard error, $e^{2} \pm E P=$ fraction of phenotypic variance due to temporary environment \pm standard error.

componentes de variância, pode superestimar o coeficiente de herdabilidade, fazendo com que fatores não-herdáveis se confundam com o componente genético aditivo.

Os resultados encontrados são consistentes com a literatura, que apresenta baixos valores de herdabilidade, indicando que grande parte das diferenças entre os animais é decorrente das diferenças de ambiente ou de fatores genéticos não-aditivos. Meyer et al. (1990) estimaram a herdabilidade para a característica DPP em animais Hereford, Angus e Europeu x Zebu e encontraram valores de 0,05; 0,08 e 0,09, respectivamente. Em outra análise da relação entre crescimento e reprodução, Meyer et al. (1991) encontraram valores de herdabilidade de 0,08 a 0,13 para as mesmas raças.

Johnston \& Bunter (1996) estimaram herdabilidade 0,12 em conjunto de dados da raça Angus. No Brasil, Pereira et al. (2000) avaliaram DPP em diversas idades e obtiveram herdabilidade 0,07 na análise que incluiu ambiente permanente e 0,17 naquela sem ambiente permanente. Esses autores relataram que DPP apresentou-se como importante característica na avaliação da fertilidade das fêmeas em todas as categorias de idade estudadas $(14,18,24$ e 36 meses e adultas). Mercadante (2001) avaliou rebanhos Nelore que passaram ou não por seleção e encontrou valores de herdabilidade de 0,02 a 0,16. Estimativas de herdabilidade para data juliana do parto, característica equivalente, variam de 0,02 a 0,17 (Azzam \& Nielsen, 1987; Meacham \& Notter, 1987; Reget \& Famula, 1993; Gressler et al., 2000).

Apesar de os componentes de variância não apresentarem grande variação com a inclusão de diferentes efeitos fixos nos modelos, os fatores de ambiente que apresentaram efeito significativo sobre DPP devem ser considerados por ocasião da análise genética dos animais. A baixa magnitude do componente genético aditivo de DPP sugere que a seleção direta deve apresentar pequenas taxas de ganho anual. No entanto, considerando a importância econômica das características reprodutivas, processos seletivos de longo prazo poderão ter impacto positivo na produtividade dos rebanhos.
Nas análises genéticas em que foram consideradas todas as fêmeas expostas à reprodução utilizando-se a penalização recomendada por Johnston \& Bunter (1996), foram encontradas estimativas de herdabilidade muito pequenas, de 0,01 e 0,07 pelos modelos com e sem o efeito de ambiente permanente, respectivamente. Como observado por Pereira (2001), o método de penalização utilizado atribui aos animais que não apresentaram registro de parição valor de DPP igual ao mais alto valor observado em seu grupo de manejo somado de 21 dias - não melhorou a identificação das diferenças genéticas entre os animais. Mercadante et al. (2002) também não detectou maior variabilidade genética ao utilizarem esse método de penalização em um conjunto de dados apenas com animais de primeira cria. Segundo Meyer et al. (1991), existem outros procedimentos mais adequados, e mais complexos, para a inclusão das fêmeas que não pariram nas análises de características reprodutivas (descritos por Carriquiry et al., 1987).

\section{Conclusões}

Fatores de ambiente, como idade da vaca no início da estação de monta, sexo do bezerro e mês do parto anterior, afetam a característica dias para o parto e devem ser considerados por ocasião da estimação dos componentes de variância da característica e da predição dos valores genéticos dos animais.

A seleção com base na característica dias para o parto pode servir para melhorar o desempenho reprodutivo de fêmeas na estação de monta, entretanto, a resposta à seleção individual poderá ser pequena .

\section{Agradecimento}

À Agropecuária Jacarezinho Ltda., pelo fornecimento dos dados, e à Fundação de Amparo à Pesquisa do Estado de São Paulo (FAPESP), pelo apoio financeiro. 


\section{Literatura Citada}

ARCHER, J.A.; ARTHUR, P.F.; PARNELL, P.F. et al. Effect of divergent selection for yearling growth rate on female reproductive performance in Angus cattle. Livestock Production Science, v.57, n.1, p.33-40, 1998.

AZZAM, S.M.; NIELSEN, M.K. Genetic parameters for gestation length, birth date and first breeding date in beef cattle. Journal of Animal Science, v.64, n.2, p.348-356, 1987.

BERGMANN, J.A.G.; GRESSLER, S.L.; PEREIRA, C.S. et al. Avaliação de fatores genéticos e de ambiente sobre diferentes características reprodutivas de fêmeas da raça Nelore em regime de estação de monta restrita. Arquivo Brasileiro de Medicina Veterinária e Zootecnia, v.50, n.5, p.633-645, 1998.

BOLDMAN, K.G.; KRIESE, L.A.; Van VLECK, D. et al. A manual for use of MTDFREML. Clay Center: USDA-ARS, 1993. 120p.

BOURDON, R.M.; BRINKS, J.S. Genetic environmental and phenotypic relationships among gestation length, birth weight, growth traits and age of first calving in beef cattle. Journal of Animal Science, v.55, n.3, p.543-549,1982.

CARRIQUIRY, A.L.; GIANOLA, D.; FERNANDO, R.L. Mixedmodel analysis of a censored normal distribution with reference to animal breeding. Biometrics, v.43, p.929-939, 1987.

FERRAZ, J.B.S.; ELER, J.P. Sumário de touros Nelore. São José do Rio Preto: CFM - Agropecuária CFM Ltda, 1998. p.10.

GAVA FILHO, J.C. Relatório final do estágio optativo do curso de Zootecnia realizado na Agropecuária Jacarezinho Ltda: bovinocultura de corte. Jaboticabal: Universidade Estadual Paulista, 1997. 43p. Relatório (Estágio para Graduação em Zootecnia) - Universidade Estadual Paulista, 1997.

GRESSLER, S.L.; BERGMANN, J.A.G.; PEREIRA, C.S. et al. Estudo das associações genéticas entre perímetro escrotal e características reprodutivas de fêmeas Nelore. Revista Brasileira de Zootecnia, v.29, n.2, p.427-437, 2000.

HOGG, R.V.; CRAIG, A.T. Introduction to mathematical statistics. 5.ed. New Jersey: Prentice Hall, 1995. 564p.

JOHNSTON, D.J.; BUNTER, K.L. Days to calving in Angus cattle: Genetic and environmental effects, and covariances with other traits. Livestock Production Science, v.45, n.1, p.13-22, 1996.

MACNEIL, M.D.; NEWMAN, S. Genetic analysis of calving date in Miles City Line 1 Hereford cattle. Journal of Animal Science, v.72, p.3073-3079, 1994.

MEACHAM, N.S.; NOTTER, D.R. Heritability estimates for calving date in simental cattle. Journal of Animal Science, v.64, n.3, p.701-705, 1987.

MERCADANTE, M.E.Z. Análise de um experimento de seleção para crescimento em bovinos Nelore: respostas direta no peso ao sobreano e correlacionadas no tamanho e reprodução das matrizes. Piracicaba: Escola Superior de Agricultura "Luiz de Queiroz", 2001. 106 p. Tese (Doutorado em Agronomia - Ciência Animal e Pastagens) - Escola Superior de Agricultura "Luiz de Queiroz", 2001.
MERCADANTE, M.E.Z.; PACKER, I.U.; RAZZOK, A.G. et al Dias ao parto de fêmeas Nelore de um experimento de seleção para crescimento: I - Modelo de repetibilidade. Revista Brasileira de Zootecnia, v.31, n.4, p.1715-1725, 2002.

MEYER, K.; HAMMOND, K.; PARNELL, P.F. et al. Estimates of heritability and repeatability for reproductive traits in Australian beef cattle. Livestock Production Science, v.25, n.1, p.1530,1990

MEYER, K.; HAMMOND, K.; MACKINNON, M.J. et al. Estimates of covariances between reproduction and growth in Australian beef cattle. Journal of Animal Science, v.69, n.9, p.35333543, 1991.

NOTTER, D.R.; JOHNSON, M.H. Simulation of genetic control of reproduction in beef cows. III. Whitin-herd breeding values estimation with known breeding dates. Journal of Animal Science, v.65, n.1, p.88-98, 1987.

NOTTER, D.R.; JOHNSON, M.H. Simulation of genetic control of reproduction in beef cows. IV. Whitin-herd breeding values estimation with pasture mating. Journal of Animal Science, v.66, n.2, p.280-286, 1988.

NOTTER, D.R.; MC FADDEN, L.G.; BERGMANN, J.A.G. Relationship between yearling scrotal circumference and measures of femeale reproduction in Angus catle. In: BEFF IMPROVEMENT FEDERATION, 25., 1993, Asheville. Proceedings... Ashevile: 1993. p.180-184.

PEREIRA, E. Análise genética de algumas características reprodutivas e de suas relações com desempenho ponderal na raça Nelore. Pirassununga: Universidade de São Paulo, 2001. 64p. Dissertação (Mestrado em Zootecnia - Qualidade e Produtividade Animal) Universidade de São Paulo, 2001.

PEREIRA, E.; ELER, J.P.; FERRAZ, J.B.S. Correlação genética entre perímetro escrotal e algumas características reprodutivas na raça Nelore. Revista Brasileira de Zootecnia, v.29, n.6, p.1676-1683, 2000.

PONZONI, R.W.; GIFFORD, D.R. Reproductive and some perinatal variables in a mixed breed beef cattle herd. Journal of Animal Breeding Genetics, v.111, n.1, p.52-64, 1994.

PRES, W.H.; FLANNERY, B.P.; TEUKOLSKY, S.A. et al. Numerical recipes. Cambridge: Cambridge University Press, 1986. $818 \mathrm{p}$.

REGET, J.E.O.; FAMULA, T.R. Factors affecting calving date and its relationship with production traits of Hereford dams. Animal Production, v.57, n.3, p.385-395, 1993.

STATISTICAL ANALYSIS SYSTEM - SAS. SAS user's guide. Version 6.12. 1998. 842p.
Recebido: 19/05/04 Aprovado: 30/01/06 